OPEN ACCESS

Edited by:

Margaret Ip,

The Chinese University

of Hong Kong,

Hong Kong

Reviewed by:

Martin Chan,

The Chinese University

of Hong Kong,

Hong Kong

*Correspondence:

Amir Abdoli

a.abdoli@modares.ac.ir,

a.abdoli25@gmail.com

Specialty section:

This article was submitted to Infectious Diseases - Surveillance,

Prevention and Treatment,

a section of the journal

Frontiers in Medicine

Received: 17 July 2017 Accepted: 15 January 2018 Published: 29 January 2018

Citation:

Abdoli A and Maspi N (2018) Commentary: Estimates of Global, Regional, and National Morbidity,

Mortality, and Aetiologies of

Diarrhoeal Diseases: A Systematic Analysis for the Global Burden

of Disease Study 2015.

Front. Med. 5:11

doi: 10.3389/fmed.2018.00011

\section{Commentary: Estimates of Global, Regional, and National Morbidity, Mortality, and Aetiologies of Diarrhoeal Diseases: A Systematic Analysis for the Global Burden of Disease Study 2015}

\author{
Amir Abdoli ${ }^{*}$ and Nahid Maspi ${ }^{2}$ \\ ${ }^{1}$ Department of Parasitology, Faculty of Medical Sciences, Tarbiat Modares University, Tehran, Iran, ${ }^{2}$ Department of \\ Parasitology, Faculty of Medical Sciences, Ilam University of Medical Sciences, Ilam, Iran
}

Keywords: systematic analysis, global burden of disease, diarrheal diseases, helminthiasis, coinfections, malnutrition

\section{A commentary on}

Estimates of global, regional, and national morbidity, mortality, and aetiologies of diarrhoeal diseases: A systematic analysis for the Global Burden of Disease Study 2015

by BD Diarrhoeal Diseases Collaborators. Lancet Infect Dis (2017) 17(9):909-48. doi:10.1016/ S1473-3099(17)30276-1

The Global Burden of Diarrheal Disease Study 2015 (GBD 2015) estimated that diarrhea was a leading cause of death and disability-adjusted life-years, especially in young children (1). Accordingly, rotavirus, Shigella spp and Salmonella spp were the leading cause of diarrhea deaths in all ages, and in children under 5 years old, rotavirus, Cryptosporidium spp., and Shigella spp. were the most causes of death (1). GBD 2015 suggested that childhood malnutrition, unsafe water, and sanitation are leading risk factors for diarrhea, particularly in sub-Saharan Africa and south Asia (1). However, the high burden of diarrheal diseases might be involved by the high burden of helminth infections, which are endemic in the same regions (2). On the one hand, helminth infections induce immune regulation toward T helper 2 and anti-inflammatory responses $(3,4)$. On the other hand, a potent Th1 immunity response and their inflammatory mediators are needed to defenses again microbial pathogens $(3,4)$. Indeed, intestinal helminths can regulate epithelial barrier function and increase epithelial permeability (5), which consequently accelerate invasion of pathogens to the intestinal barriers. Hence, helminth coinfections suppress immune responses against microbial pathogens and increase susceptibility and severity of infectious diseases, including HIV, malaria, and tuberculosis, which are the major causes of morbidity and mortality in sub-Saharan Africa and south Asia $(6,7)$. Helminth coinfections also increase colonization and intensity of intestinal pathogens $(8,9)$. In this regard, Harris et al. (10) observed that Vibrio cholerae infected patients who coinfected with helminths had lower antibody response to cholera toxins. In the case of Salmonella and schistosomiasis coinfections, both organisms synergically induce immunological alterations that lead to increased disease duration of both infections. Importantly, Schistosomia can act as a latent carrier of Salmonella, and Salmonella is able to persist in the helminth's tegument and protected from antibacterial drugs (8). In murine models, Su et al. (11) found that coinfections of Heligmosomoides polygyrus and Salmonella Typhimurium led to poor control of bacterial replication 
and increased intestinal inflammation in coinfected animals than animals infected with $S$. Typhimurium alone. Su et al. (11) also reported that coinfected animals had reduce in the recruitment of neutrophils and reduced in expression of chemoattractants CXCL2/macrophage inflammatory protein 2 and CXCL1/ keratinocyte-derived chemokine in the site of Salmonella infection (11). Bobat et al. (12) demonstrated that coinfection with Nippostrongylus brasiliensis decrease protective immunity during natural infection or immunization with Salmonella Typhimurium in a murine model. Reynolds et al. (9) found that $H$. polygyrus coinfection enhance colonization and virulence of Salmonella by disruption of intestinal metabolome. Coinfection with $H$. polygyrus also impaired autophagy-mediated property of macrophages to killing the enteropathogen Citrobacter rodentium in a mouse model (13). Bednarska et al. (14) investigated the interaction of Heligmosomoides bakeri and Cryptosporidium parvum coinfection in C57BL/6 mice. They found that helminth coinfection led to prolonged course and intensity of $C$. parvum infection (14). Reese et al. (15) found that helminth infection reactivated latent $\gamma$-herpesvirus infection in a murine model via induction of anti-inflammatory cytokine interleukin-4 (IL-4) and the activation of the transcription factor STAT6. Reese et al. also demonstrated that IL-4 blocked the antiviral effects of interferon- $\gamma$ and promoted viral replication (15). Osborne et al. (16) found that concurrent of helminths with murine norovirus infection impaired antiviral immunity alongside with changes in the intestinal microbiota. Interestingly, they observed that helminth induce immunomodulation and impaired antiviral

\section{REFERENCES}

1. BD Diarrhoeal Diseases Collaborators. Estimates of global, regional, and national morbidity, mortality, and aetiologies of diarrhoeal diseases: a systematic analysis for the Global Burden of Disease Study 2015. Lancet Infect Dis (2017) 17(9):909-48. doi:10.1016/S1473-3099(17)30276-1

2. Havelaar AH, Kirk MD, Torgerson PR, Gibb HJ, Hald T, Lake RJ, et al. World Health Organization global estimates and regional comparisons of the burden of foodborne disease in 2010. PLoS Med (2015) 12(12):e1001923. doi:10.1371/journal.pmed.1001923

3. Hotez PJ, Brindley PJ, Bethony JM, King CH, Pearce EJ, Jacobson J. Helminth infections: the great neglected tropical diseases. J Clin Invest (2008) 118(4): 1311-21. doi:10.1172/JCI34261

4. Maizels RM, McSorley HJ. Regulation of the host immune system by helminth parasites. J Allergy Clin Immunol (2016) 138(3):666-75. doi:10.1016/ j.jaci.2016.07.007

5. McKay DM, Shute A, Lopes F. Helminths and intestinal barrier function. Tissue Barriers (2017) 5(1):e1283385. doi:10.1080/21688370.2017.1283385

6. Salgame P, Yap GS, Gause WC. Effect of helminth-induced immunity on infections with microbial pathogens. Nat Immunol (2013) 14(11):1118-26. doi:10.1038/ni.2736

7. Abdoli A, Pirestani M. Are pregnant women with chronic helminth infections more susceptible to congenital infections? Front Immunol (2014) 5:53. doi:10.3389/fimmu.2014.00053

8. Hsiao A, Toy T, Seo HJ, Marks F. Interaction between Salmonella and schistosomiasis: a review. PLoS Pathog (2016) 12(12):e1005928. doi:10.1371/ journal.ppat.1005928

9. Reynolds LA, Redpath SA, Yurist-Doutsch S, Gill N, Brown EM, van der Heijden J, et al. Enteric helminths promote Salmonella coinfection by altering the intestinal metabolome. J Infect Dis (2017) 215(8):1245-54. doi:10.1093/infdis/jix141 immunity even in germ-free mice, which indicated a microbiotaindependent mechanism of immunomodulation (16). Taken together, helminth coinfections can augment diarrheal diseases via increased susceptibility and severity of intestinal pathogens.

GBD 2015 also discussed that, "malnutrition or regular illness during the first few years of life has negative effects on future cognitive development, education, and productivity". It is well documented that malnutrition is an important cause of immune suppression, which consequently enhance susceptibility and severity to intestinal pathogens (17). On the other hand, chronic helminth infections are linked to different insidious persistent health conditions such as protein-calorie undernutrition, anemia, growth stunting, and fatigue (3). Chronic helminth infections are also linked to poor cognitive development in children $(3,18)$. Thus, helminth infections could have indirect negative effects on future cognitive development, education, and productivity of children.

In conclusion, helminth coinfections might be a neglected risk factor for control of diarrheal diseases due to intestinal pathogens, so control and treatment of helminths may help to control diarrheal diseases due to microbial pathogens. Although, randomized trials are needed to demonstrate the effects of helminth coinfections on diarrheal diseases.

\section{AUTHOR CONTRIBUTIONS}

AA and NM conceived and designed the manuscript. AA wrote the manuscript.

10. Harris JB, Podolsky MJ, Bhuiyan TR, Chowdhury F, Khan AI, LaRocque RC, et al. Immunologic responses to Vibrio cholerae in patients co-infected with intestinal parasites in Bangladesh. PLoS Negl Trop Dis (2009) 3(3):e403. doi:10.1371/journal.pntd.0000403

11. Su L, Su C-W, Qi Y, Yang G, Zhang M, Cherayil BJ, et al. Coinfection with an intestinal helminth impairs host innate immunity against Salmonella enterica serovar Typhimurium and exacerbates intestinal inflammation in mice. Infect Immun (2014) 82(9):3855-66. doi:10.1128/IAI. 02023-14

12. Bobat S, Darby M, Mrdjen D, Cook C, Logan E, Auret J, et al. Natural and vaccine-mediated immunity to Salmonella Typhimurium is impaired by the helminth Nippostrongylus brasiliensis. PLoS Negl Trop Dis (2014) 8(12):e3341. doi:10.1371/journal.pntd.0003341

13. Su C-W, Cao Y, Zhang M, Kaplan J, Su L, Fu Y, et al. Helminth infection impairs autophagy-mediated killing of bacterial enteropathogens by macrophages. J Immunol (2012) 189(3):1459-66. doi:10.4049/jimmunol.1200484

14. Bednarska M, Bajer A, Sinski E. Cryptosporidium parvum: the course of Cryptosporidium parvum infection in C57BL/6 mice co-infected with the nematode Heligmosomoides bakeri. Exp Parasitol (2008) 120(1):21-8. doi:10.1016/j.exppara.2008.04.007

15. Reese T, Wakeman B, Choi H, Hufford M, Huang S, Zhang X, et al. Helminth infection reactivates latent $\gamma$-herpesvirus via cytokine competition at a viral promoter. Science (2014) 345(6196):573-7. doi:10.1126/science. 1254517

16. Osborne LC, Monticelli LA, Nice TJ, Sutherland TE, Siracusa MC, Hepworth MR, et al. Virus-helminth coinfection reveals a microbiotaindependent mechanism of immunomodulation. Science (2014) 345(6196): 578-82. doi:10.1126/science.1256942

17. Cunningham-Rundles S, McNeeley DF, Moon A. Mechanisms of nutrient modulation of the immune response. J Allergy Clin Immunol (2005) 115(6): 1119-28. doi:10.1016/j.jaci.2005.04.036 
18. Owada K, Nielsen M, Lau CL, Clements ACA, Yakob L, Soares Magalhães RJ. Measuring the effect of soil-transmitted helminth infections on cognitive function in children: systematic review and critical appraisal of evidence. Adv Parasitol (2017) 98:1-37. doi:10.1016/bs.apar.2017.05.002

Conflict of Interest Statement: The authors declare that the research was conducted in the absence of any commercial or financial relationships that could be construed as a potential conflict of interest.
The reviewer MC and handling editor declared their shared affiliation.

Copyright (C) 2018 Abdoli and Maspi. This is an open-access article distributed under the terms of the Creative Commons Attribution License (CC BY). The use, distribution or reproduction in other forums is permitted, provided the original author(s) and the copyright owner are credited and that the original publication in this journal is cited, in accordance with accepted academic practice. No use, distribution or reproduction is permitted which does not comply with these terms. 